


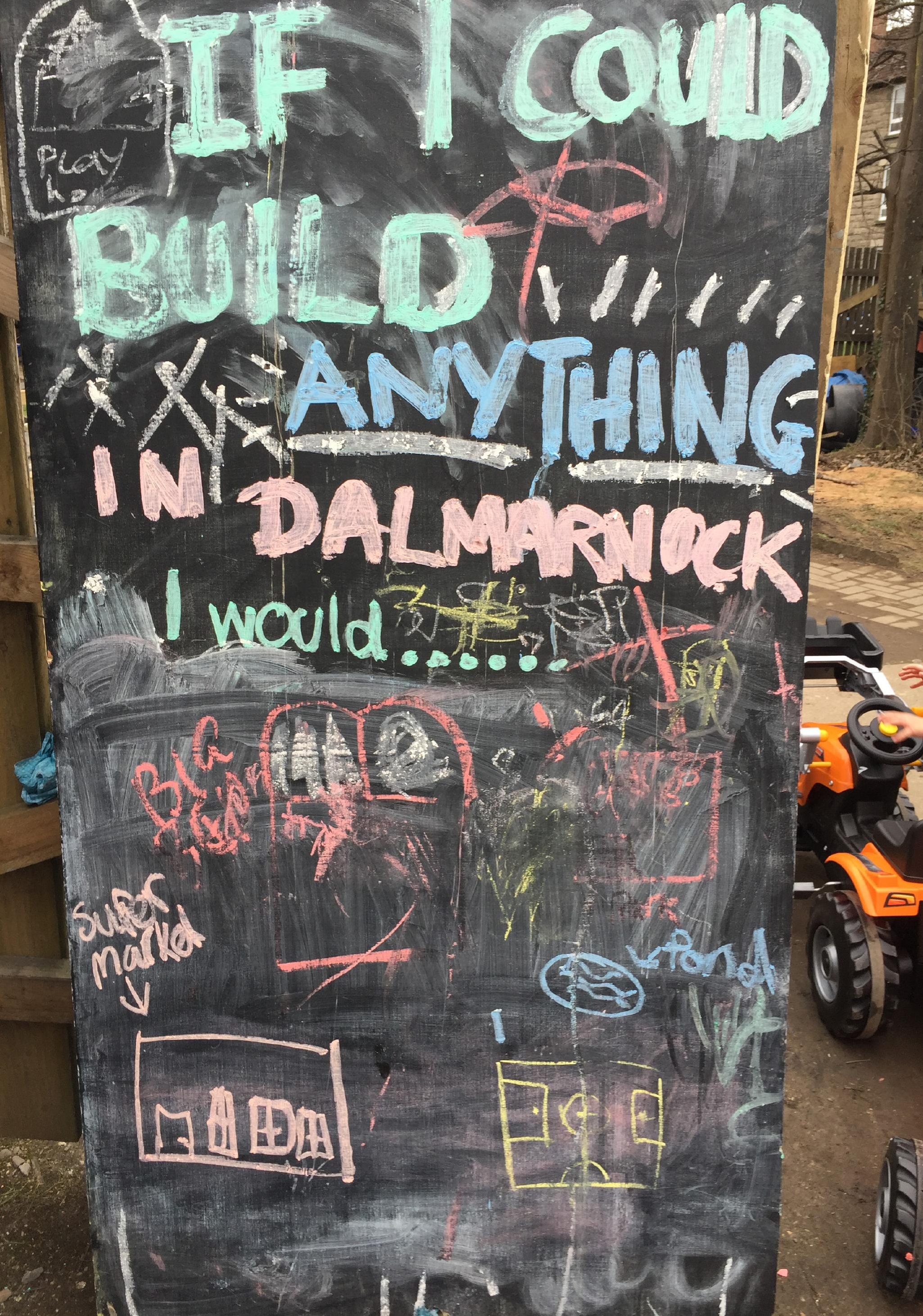




\section{Project Summary}

The community food hub project at the Baltic Street Adventure Playground (BSAP) in Dalmarnock, Glasgow, established interconnected community food work streams including surplus distribution, growing and community meals; building on the strong foundation of the existing food work carried out by BSAP. The project provided an important space to reflect on food and sustainability from the perspective of an area of urban deprivation in Glasgow's East End. This report outlines the findings of our research with BSAP between January 2019 and January 2020. Learning together with BSAP and the local residents of Dalmarnock, the project identified two key themes around sustainability within this context. These are the deep roots of food culture and the importance of connecting sustainability to food access as a form of food justice. The findings of this project highlight the importance of community ownership and governance, and of building sustainability from the bottom up. Starting from the perspective of Dalmarnock and working from the needs and experiences of BSAP's community, the project also points to the potential blind spots in current sustainability policy and reiterates the imperative to ensure that no one is left behind.

\section{Acknowledgements}

This project is a collaboration between University of Glasgow and Baltic Street Adventure Playground and was funded by EU/Scottish Government Social Innovation Funding Stage 2.

\section{Baltic Street Adventure Playgoround
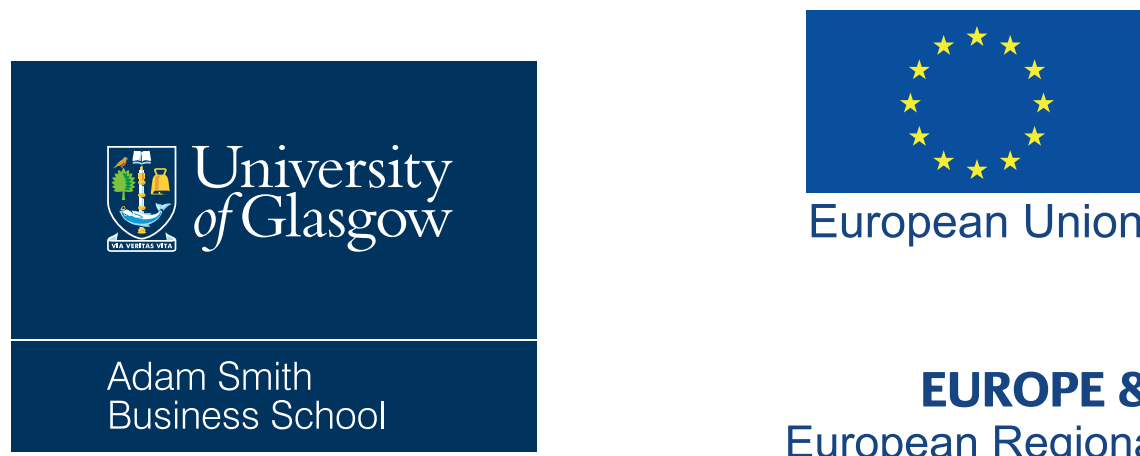 \\ European Union

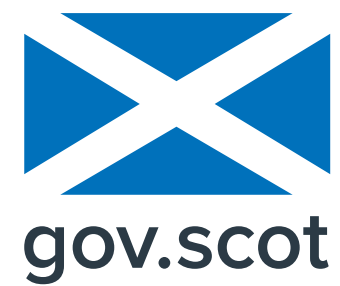 \\ EUROPE \& SCOTLAND \\ European Regional Development Fund Investing in a Smart, Sustainable and Inclusive Future}

The images on pages 6, 9, 12, 14, 15 and 17 are credited to Propagate (www.propagate.org.uk). 


\section{Authors}

Dr Helen Traill

Helen.Traill@glasgow.ac.uk

Professor Deirdre Shaw*

Deirdre.Shaw@glasgow.ac.uk

Dr Stephanie Anderson

Stephanie.Anderson@glasgow.ac.uk

* Corresponding author

\section{Professor Andrew Cumbers}

Andrew.Cumbers@glasgow.ac.uk

Professor Robert McMaster

Robert. McMaster@glasgow.ac.uk

\section{Natalie Marr}

Natalie.Marr@glasgow.ac.uk

\section{Contents}

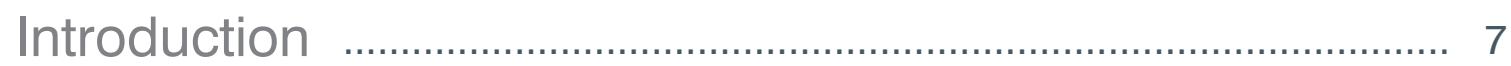

Why thinking about sustainability is important in ................................ 8 an area of urban deprivation

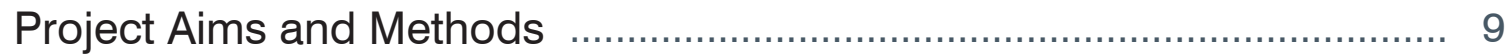

Working Alongside, Learning Together f.............................................. 9

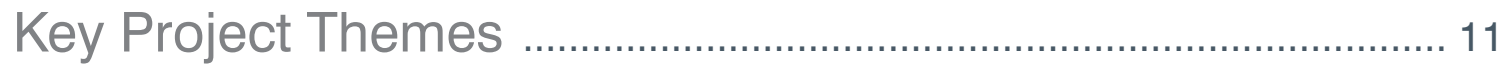

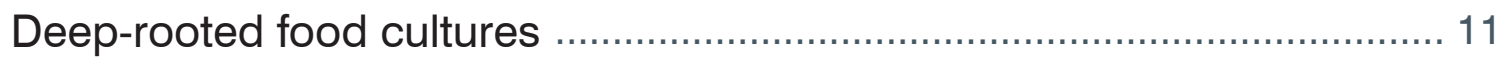

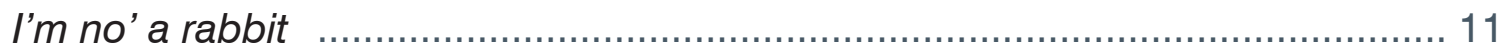

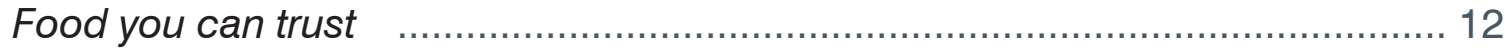

Opening conversation spaces $\quad$............................................................. 13

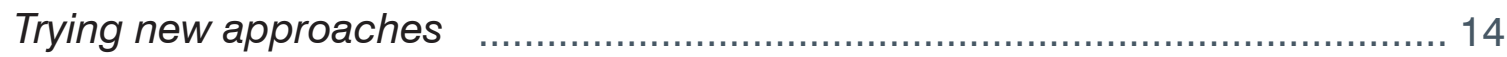

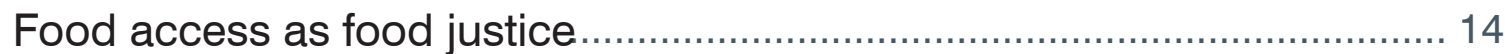

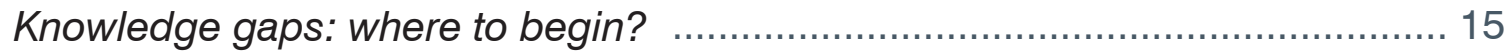

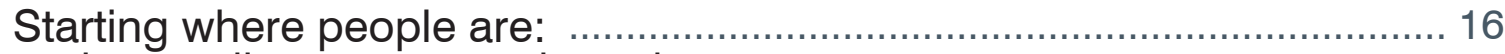

understanding access and need

Discussion and Conclusions ......................................................... 18

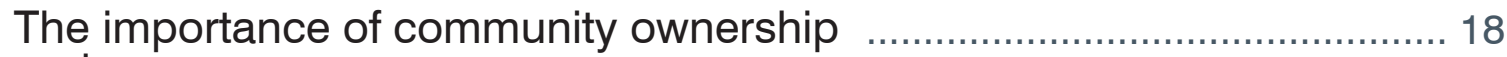
and governance

Building from joy and the immediate moment $\quad$....................................... 18

Facilitating community ownership $\quad$..................................................... 19

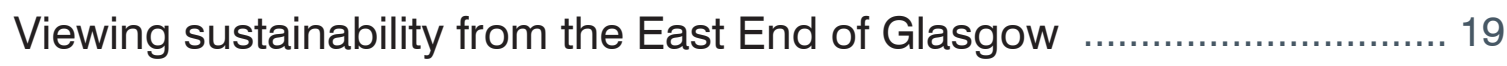

Sustainability from the ground-up $\quad$..................................................... 19

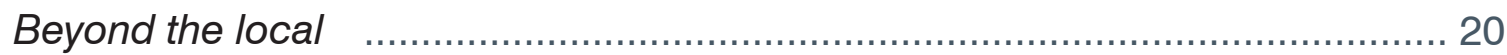




\section{Introduction}

Baltic Street Adventure Playground (BSAP) was established in 2013 in Dalmarnock, Glasgow, as a response to a lack of children's play facilities in the area. BSAP is community-focused and child-led; children are at the heart of decision making from structuring their own play to the day-to-day management of the site's amenities

Following the Commonwealth Games in $2014,{ }^{1}$ as a result of the clearance of buildings to host the event, Dalmarnock experienced the reduction and even closure of many of its existing retail and community facilities. As part of this, the area was hit by a serious reduction in food retailing outlets. It was recently ranked as Scotland's 'most deprived food desert' ${ }^{2}$ and is located in the top $5 \%$ of measures of multiple deprivation $^{3}$. In short, Dalmarnock is as a place of spatial vulnerability, where disadvantages are intensified due to a lack of essential amenities locally ${ }^{4}$. The demolition of the community centre, housing and shops to make space for the Commonwealth Games Athletes Village and the closure of a multimillion pound replacement community facility in January $2019^{5}$ has left the area with a legacy of failed top-down regeneration efforts.

Against this backdrop of urban restructuring, multiple deprivation and spatial vulnerability, BSAP is a space initiated from within the community that plays an important role in providing much needed play space for children, rebuilding social connections and providing meals for families. This project, in collaboration with BSAP, aimed to support the establishment of a community food hub. This was achieved via a bottom-up approach informed by BSAP's existing ethos of 'learning alongside' those who attend and specifically its commitment to working democratically to co-produce the space with local children.

Users of BSAP span multiple groups and geographies, from immediate neighbours of the space to summer visitors from as far afield as Inverness and Birmingham. Broadly, daily users fall into two (sometimes overlapping) categories, namely, residents of Dalmarnock and those with children (either as parents or often from nurseries or childcare groups). During school holiday periods, numbers increase significantly as visitors from across the city and beyond attend BSAP.

\footnotetext{
1 An excellent website and critical assessment of the negative impact of the Games on the wider East End area was established by the group Games Monitor. See: https://gamesmonitor2014.org/. One academic paper has referred to the Games process as one that has effectively been 'to violently displace working class neighbourhoods'. See: Gray, N. and Porter, L. (2014). By Any Means Necessary: Urban Regeneration and the "State of Exception" in Glasgow's Commonwealth Games 2014. Antipode 47 (2): pp. 380-400.

2 Corfe, S. (2018). What are the Barriers to Eating Healthily in the UK? London, Social Market Foundation, p. 28.

3 Scottish Index of Multiple Deprivation, https://simd.scot/2016/, [last accessed 20 April 2020].

4 Saatcioglu, B. and Corus, C. (2016). Exploring spatial vulnerability: inequality and agency formulations in social space. Journal of Marketing Management 32 (3-4), pp.230-251.

5 Hutcheon, P. 'Commonwealth games legacy project closes after financial problems'. The Herald. 27 January 2019. Available: https://www.heraldscotland.com/news/17388033.commonwealth-games-legacy-project-closes-after-financial-problems/ [Last accessed 20 April 2020]
} 
The focus of this project was on regular BSAP users, who typically live in the local community, although one does not have to be from Dalmarnock to benefit from the project. This community (in the descriptive geographical sense ${ }^{6}$ ) sits at the core of the BSAP project and is focused around the local children, whose play and self-organisation forms the focus of BSAP's work. Inevitably, there is a fine balance to be maintained between benefiting their local community and offering an inclusive, welcoming play space. The community food hub project greatly enhanced BSAP's ability to support community food growing, skills development and integration of sustainable practices across their various activities, but particularly within this core community group.

\section{Why thinking about sustainability is important in an area of urban deprivation}

It is important to consider sustainability in collaboration with organisations such as, BSAP in order to open up the interaction between sustainability, deprivation and spatial vulnerability. We take a cue from Rebecca Elliot's work that asks us to consider climate change from the frame of loss. Narratives of sustainability are often framed in terms of harmony and holism; an attention to loss - as disappearance, destruction, dispossession and depletion ${ }^{7}$ - raises questions, such as: what can we ask people living in areas of multiple deprivation and spatial vulnerability to lose from already straitened lives? How we can formulate more just visions of sustainability that are sensitive to the specific contexts in which we are working? Building effective practices of sustainability in spatially vulnerable places needs to be produced with the communities themselves and to recognise both the limitations, but also the potential and diversity, of being attentive to local contexts.

Within this, community food can be a key platform for exploring sustainability horizons. Community food is much more than a shared meal; it has potentially important social, health and wellbeing properties in connecting people and opening up conversations about the food system ${ }^{8}$. This project has taken a wide-angled lens on community food, encompassing food growing, community meals, distributing food surplus provided through FareShare deliveries ${ }^{9}$, and the many afternoons of feeding children at BSAP as the basis for this exploration. We, thus, recognise a community food hub as a multi-purpose space that unites the connective power of food across a variety of forms, tailored to the needs of its location, and the social importance of that connection. This builds on scholarly work that recognises community as a verb - emerging in on-going connections between people ${ }^{10}$.

6 While we recognise community is a complicated term that does not make a useful analytical concept (Traill 2018), it is used descriptively both within BSAP and here to refer to both the local people of Dalmarnock and, more specifically, the users of the BSAP project. See Traill, H. (2018) Community as idea and community practices: tensions and consequences for urban communal growing in Glasgow, PhD Thesis, London School of Economics and Political Science.

7 Elliot, R. (2018). The Sociology of Climate Change as a Sociology of Loss. European Journal of Sociology, 59, no. 3, pp. $301-337$.

8 See, e.g.: Marovelli, B. (2019). Cooking and eating together in London: Food sharing initiatives as collective spaces of encounter', Geoforum, 99: pp. 190-201; Traill, H. (2018)

9 FareShare are a non-profit who collect surplus from supermarkets, wholesalers and supply chains and distribute it via a membership scheme to community organisations and charities (https://fareshare.org.uk/what-we-do/).

10 Rogaly, B. (2016). 'Don't show the play at the football ground, nobody will come': the micro-sociality of co-produced research in an English provincial city Sociological Review. 64 (4), pp. 657-680. Studdert, D. and Walkerdine, V. (2016). Rethinking Community Research. Basingstoke: Palgrave. 
This project, thus considered the interrelationships between community food and sustainability. From this framework, we understand the community food hub as a space of exploration and possibility, and as a vital node for rebuilding sociality and strengthening community within Dalmarnock.

\section{Project Aims and Methods}

Working collaboratively with BSAP between January 2019 and January 2020, the project's overarching practical aim was to facilitate the establishment of a community food hub in one of Scotland's most deprived areas; a district with few opportunities to access fresh food. This built on the strong foundation of existing food work at BSAP. As part of this, the food hub would promote:

- Social inclusion through a bottom-up approach established by BSAP. The hub is community-focused, enabling children and adults access to guidance on food growing, healthy food and access to fresh produce.

- Promotion of environmental sustainability through, for example, the use of recycled materials, reduction of waste, organic food growing and composting.

\section{Working Alongside, Learning Together}

BSAP is a child-led play facility where trained staff provide food and support for the children. At the heart of BSAP's culture is the idea of 'play first'. Children play and learn informally through cooperation and trust. BSAP's mission is to support incremental, ground-up activities. The project aims were achieved by building on existing trust relationships from earlier collaborations and working alongside BSAP and the community.

BSAP is valued by the local community and plays an important role in facilitating networks of mutual support across the East End of Glasgow. Residents have variously described it as a 'focal point', 'a community centre' and 'the heart and soul of Dalmarnock'. It, therefore, represents an important space for potentially transformative social interactions around food and sustainability learning and, as a result, can offer insights to similar organisations and initiatives across Glasgow and beyond.

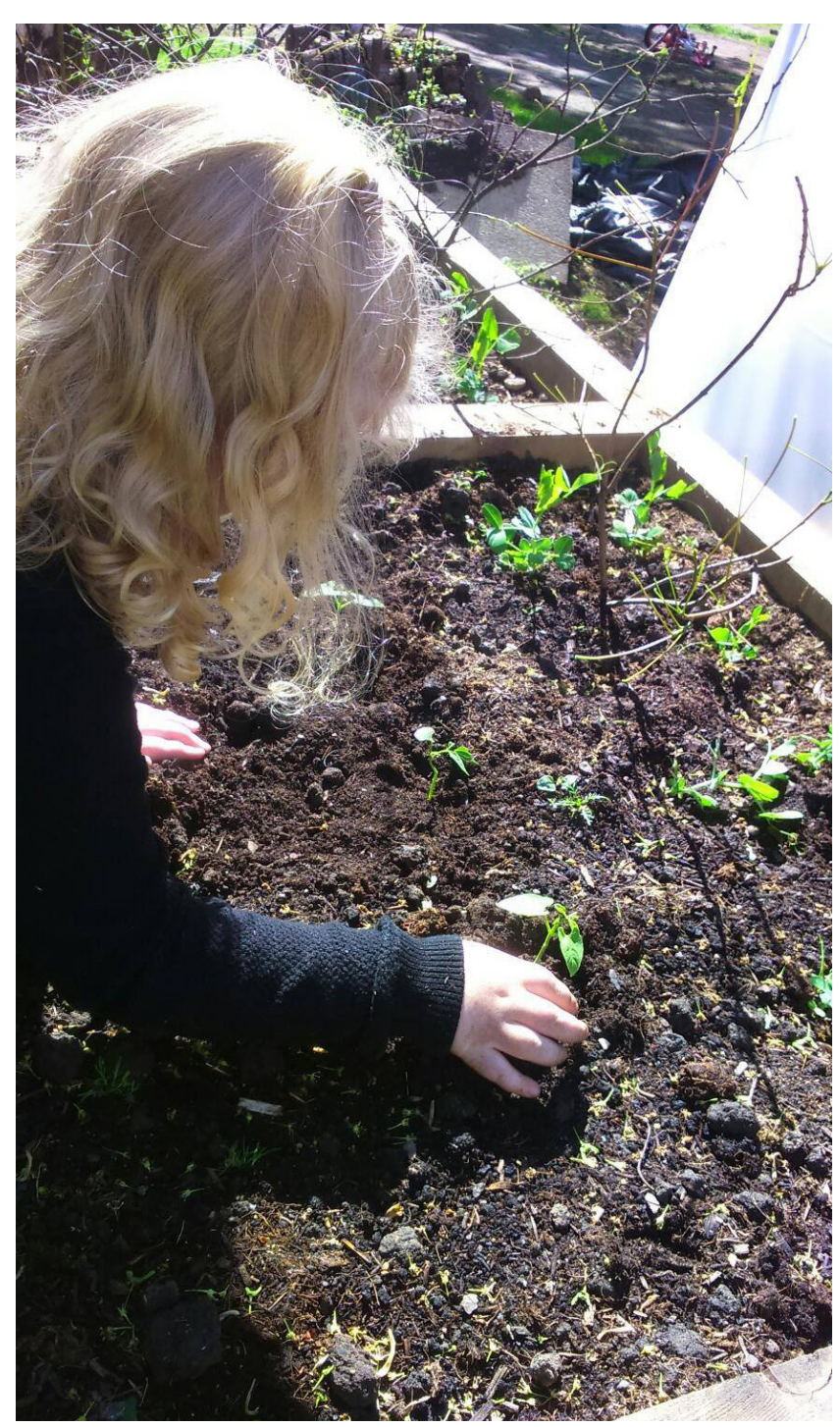


To facilitate greater knowledge and experience of food production, the project employed an experienced community gardener who supported and advised on growing and animal welfare. This afforded the local community the opportunity to access food production activities via communal learning. In doing so, participants could reflect on horticultural practices and gain insight and knowledge of environmental sustainability through discussions on composting, disposables, recycling and waste.

The project has been critical in helping BSAP develop its capacity and expand its community food hub activities through supporting a sustained consideration of the site's environmental impact and potential. Beyond the financial investment in assets and infrastructure, such as, a minibus and a polytunnel for the garden, BSAP has emphasised the value of the collaborative process that allows for dialogue and exchange between BSAP staff, children, parents and expert practitioners:

As an organisation we can be quite focused on the day to day, so considering broader issues often gets put on the backburner. The project kept us focused on issues of sustainability, which gave us a reason to keep coming back to it. (BSAP Playworker $1^{11}$ ) 


\section{Key Project Themes}

From our collaborative experience we highlight two key themes for discussion below, namely, deeprooted food cultures, and food access as food justice.

In the broader context of sustainability discussions, where diets are a contested terrain, these insights have important ramifications. The $\mathrm{FAO}^{12}$ and others have highlighted the need to shift towards healthier and more sustainably produced food, which includes a move to more plant-based diets ${ }^{13}$. Our insights suggest that community food hubs can play an important role in engendering this shift but in ways that also promote dignity and care in relation to working with and producing food, thus, having the potential to contribute towards a more just transition ${ }^{14}$.

\section{Deep-rooted food cultures}

\section{'I'm no' a rabbit'}

Deep-rooted localised food cultures tended to be most noticeable in relation to vegetables and a vegetable-based diet. Such foods were not considered a 'normal' part of the diet for many BSAP users. For some, this was expressed in terms of suspicion or dismissal; as one long-term, older, female Dalmarnock resident put it more than once, 'I'm no' a rabbit'. For others, this was expressed as discomfort - although not necessarily an unwillingness to engage - brought about by a lack of knowledge as to how to eat or cook certain foods. This was particularly evident with some of the less familiar surplus supplied by FareShare.

This was also a challenge in terms of establishing the garden. One of the BSAP playworkers described how the produce that was grown did not always reflect where people were at with their tastes and food experiences:

[...] but to grow kale, I think it's just a quantum leap too far at the beginning. Yes, that will come down the line but growing loads and loads of lettuce and salad produce [...] people would come and take one wee bit of lettuce to have with their food. Now, it's one wee bit of lettuce more than they may normally have had but [...] I think we could have maximised the beds to produce more produce that would have been more palatable at the beginning, if that's the right expression, but there would have been more of an uptake on the yield from it. (BSAP Playworker 2)

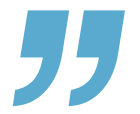

Tailoring community growing to a space is an iterative process and learning from this initial food hub project will be adapted for future years. As the above quote suggests, it is critical that food growing in the first instance is mindful of existing food experiences, while recognising the importance of gradually introducing new types of food and ways of preparing and cooking. Attitudes as to what constitutes 'good food' suggest a great deal of subjective variability. 
Important in this context is an understanding of 'good food' over which local communities have ownership and control. Most definitions of sustainable food suggest food should be healthy and sustainably-sourced, but also accessible and culturally appropriate to particular local contexts. ${ }^{15}$ Balancing these different aspects is not straightforward, and the experience from BSAP suggests that food cultures need to be centred in ongoing community food work and practices.

It is important to build new and diverse food experiences from existing behaviours, rather than attempting to impose idealised sustainable food practices from outside. As we detail below, this worked best at BSAP, through the support of key individuals, particularly the playworker with responsibility for food who could play an intermediary role in introducing new types of food and ideas about healthier food consumption and preparation.

\section{Food you can trust}

BSAP are trusted purveyors of food and survey data consistently showed that people regarded the food favourably. In surveys undertaken after the October holiday programme in 2019 , for example, $97.7 \%$ of responses praised the food offered at BSAP.

Much of the trust vested in BSAP in relation to food centres around the playworker with responsibility for food, who is well known and liked. One lunchtime during the summer programme, as the queues stretched across the playground and children and families waited to try one of the many dishes on offer from the tiny BSAP kitchen, this appreciation was made vocal. Normally one to stay out of the limelight, the playworker appeared from the kitchen with crockery for serving food, only to be met with three rowdy cheers from the crowd, led by another playworker.

Seeing the playworker prepare meals and having an opportunity to chat with him about the food they are eating, gives people the confidence to try something different and explore new relationships with food. BSAP know their audience well and have established trust over time. This has facilitated conversations around food in a way that is sensitive to people's needs and interests, whilst gently introducing new ideas. Reflecting on her introduction to a new cheese, one older local resident with grandchildren told us:

\section{Then I got cheese... [I]t was that nice,} you kept thinking about what could I make that I could use that cheese and everything revolved around [it]... But a lot of things I've had out of there I've never, I wouldn't even have tried if I hadn't of tasted them in there and I buy them now.
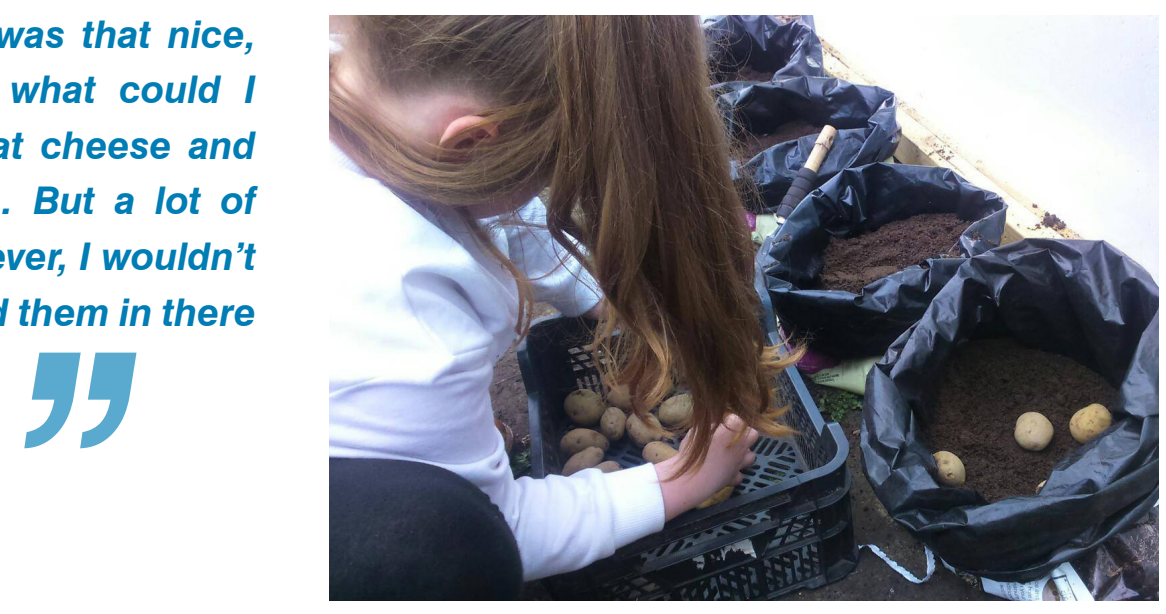

15 'Sustainable diets are those diets with low environmental impacts, which contribute to food and nutrition security and to healthy life for present and future generations. Sustainable diets are protective and respectful of biodiversity and ecosystems, culturally acceptable, accessible, economically fair and affordable; nutritionally adequate, safe and healthy; while optimizing natural and human resources.' (FAO 2012), Sustainable Diets and Biodiversity. Available: http://www.fao.org/3/a-i3004e.pdf [last accessed 20 April 2020] 
BSAP are a trusted source of good food and information about how to use some of the more or less familar items that arrive through the FareShare scheme. Working to support and build on this trust, we saw through the project a reduction in the waste of otherwise edible food that arrives at BSAP, and an opening up of new food experiences for the BSAP community.

\section{Opening conversation spaces}

As noted above, conversations about food regularly took place at BSAP. Growing activities provided a key space for this. Through this project, people have tried new foods that have been grown on site, discussed composting and food waste, as well as the pleasure of eating home-grown food. For some, the BSAP food takes them miles away, to countries they have emigrated from or to more rural homes. As one parent who regularly attends BSAP put it:

And, to be honest, you see the eggs, they're different, the taste is different from the one we got from the supermarket, and it just reminds me back, like back home, you get the real taste of the eggs, like newly laid eggs and you know...so it's good.

Conversations around food happen informally, whether it is children talking to their caregivers about the food they are eating or adults swapping food stories about things they have discovered through FareShare. ${ }^{16}$

The following is an example of such an exchange during a group interview :

Anne: Yeah, uhuh. Fish, it looks like a pastry thing.

Lindsey: I ate that this morning for my breakfast! [laughing]

Anne: It was so nice! And I would never buy that.

Stacey: It was fish with parsley and cheese, weren't it?

Anne: $\quad$ Then it was, in pastry. Different things with curry sauce, that's what I've tried. Coconut milk, things like that. I haven't used that yet, but I'm going to $[\ldots]$

Stacey: That fish thing, I'm definitely going to go buy that again.

Through these conservation spaces, informal learning takes place and local people can bond over the everyday sociality of a shared food culture. This also affords the normalisation of surplus food and encourages people to use it and experiment with ideas and recipes that are new to them. 


\section{Trying new approaches}

Despite reservations about some of the food, the trust and rapport built up between BSAP and its community means that people are willing to try new things and explore new relationships with food. This might include trying a bit of salad at lunchtime or pulling a fully-grown carrot out of the soil and being able to eat it almost immediately.

In keeping with BSAP's ethos of 'play first', new approaches are best introduced when they are curious and joyful rather than overly formal. As one playworker put it:

So a lot of the outdoor stuff's about planning in the moment and like taking dialogue and leadership from the children and their own voices (BSAP Playworker 4)

In this way, the community food hub was tailored to the existing dynamics of the site. Whilst behavioural change is not a priority for BSAP, their inclusive and non-punitive approach has helped prompt reflection and changes in users' foodways and entrenched attitudes to food:

[T]hat's when the critical learning takes --- they find it from us, and from other people that are working here, they're learning without actually knowing they're learning, which is the best way to do it, because then they enjoy it. (BSAP Playworker 3)

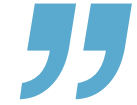

In this context, change has occurred incrementally through small moments of exchange and discovery in ways that feel of the space rather than imposed.

One child who was keen to be involved in the food growing developed such an interest in the process that he declared he wanted to be a farmer when he grew up. A field trip facilitated by the project to a local market garden allowed him to meet urban vegetable farmers and try out their tools. In this way, his notion of a farm as a place of livestock and countryside was gently challenged and his interest in growing encouraged. For him, the opening up of this alternative career path as a real, urban possibility demonstrates the longer-term possibilities of the space, in training a next generation of horticultural and agricultural workers in a place not traditionally associated with food growing.

\section{Food access as food justice}

The project highlighted how access to food presented issues of justice. This emerged as knowledge gaps and the need to learn alongside local people, to create a community food hub that is not only used by locals but gives them a sense of ownership.

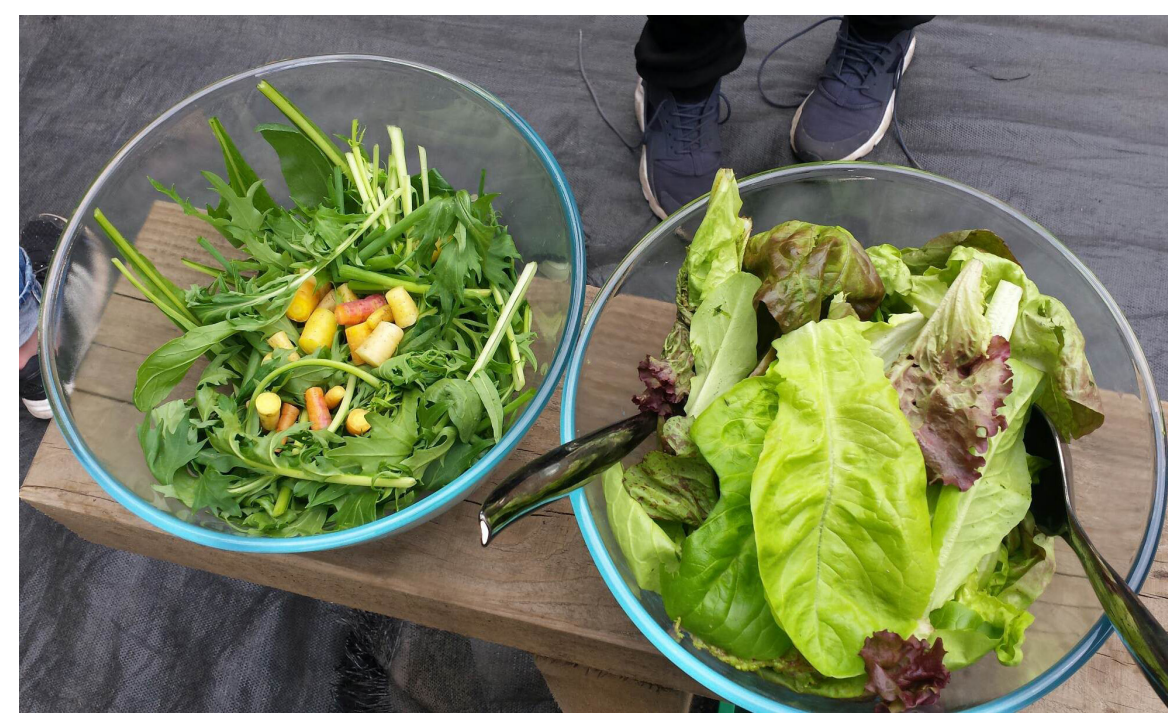




\section{Knowledge gaps: where to begin?}

While there has been a lot of interest in the garden, a lack of knowledge has posed a barrier to getting the full range of benefits from the growing season. Drawing on the knowledge and expertise of the project's community gardener, was key in distributing knowledge between the BSAP team and users of the space. A BSAP Playworker reflected on the garden prior to the project:

\section{We sought funding to establish the raised beds that you see now so we'd the raised beds} in, but we really hadn't thought about well who's the knowledge, what to put in this apart from soil and a bunch of seeds which we'd done before and you've got all sorts growing within each other, it's not getting thinned out, it's not savvy, so the function of growing a load of seeds in a bottle and then watching something sprout out of it, it's fine, but there's nothing meaningful in terms of a harvest from that. (BSAP Playworker 2)

Thus, growing activities have provided learning for local people, but also for the staff themselves. However, for locals it was not always clear how to get involved and many assume that they need to be skilled and experienced to participate. Some gardening tasks like moving large quantities of compost are not always suitable for local children, limiting the conversations that could be taking place around growing.

There are also temporal challenges to addressing knowledge gaps. Our community gardener noted a marked difference during the holiday period when attendance increased significantly, sometimes to an excess of 500 people a day. Such a volume makes meaningful interaction with more regular local people a challenge. This meant that the local community missed out on a more focused food growing programme during an important stage of plant growth development, creating discontinuity in learning.

A challenge for BSAP is learning how to convert expressed interest in the garden into tangible activities and sustained engagement. The project partnership and funding has allowed flexibility to explore and learn from mistakes, such as planting too many tomato plants or growing food that does not reflect the current tastes of the community.

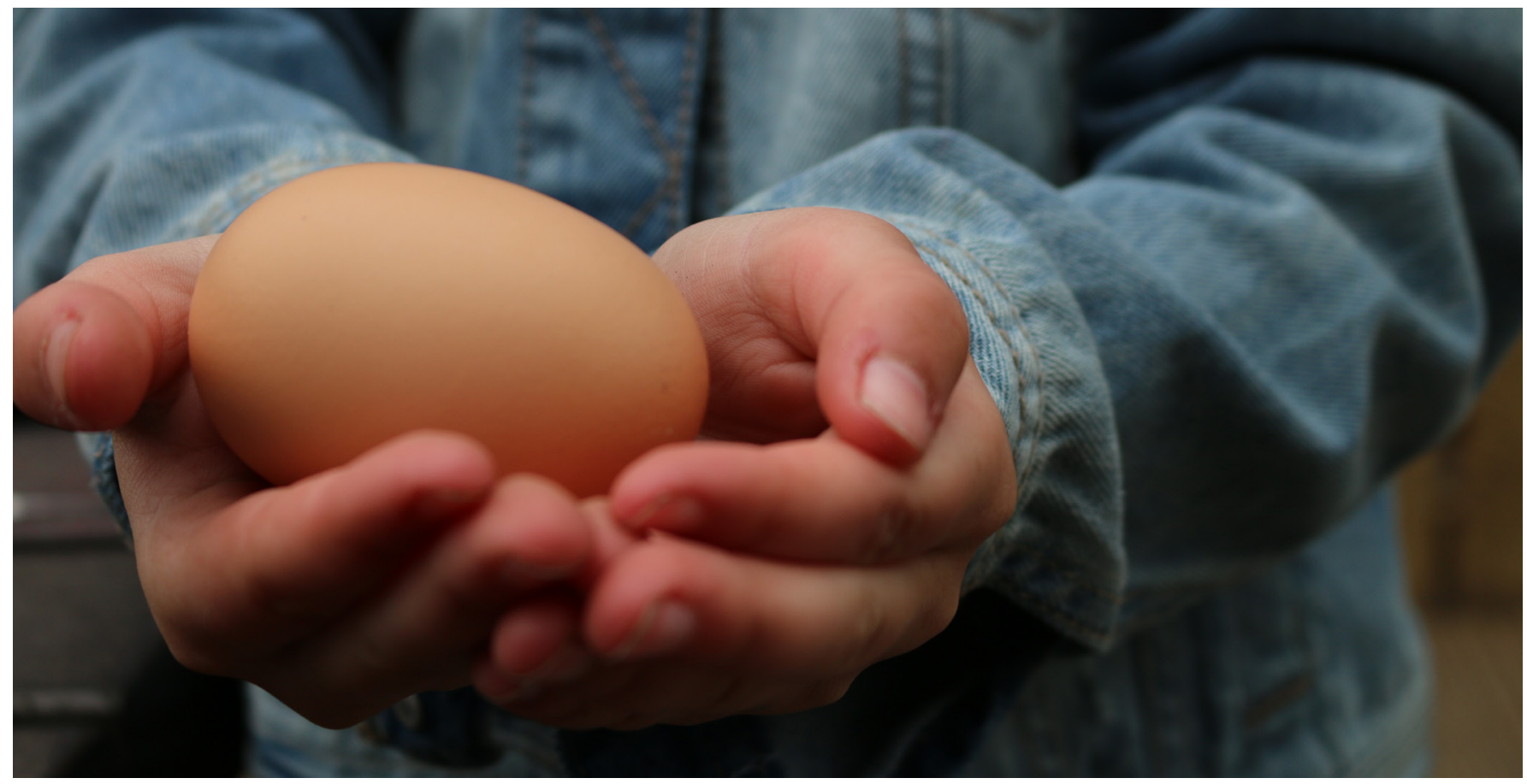




\section{Starting where people are: understanding access and need}

\section{Baltic Street are very, very good at engaging the community, very good at understanding the community's need at that moment. (BSAP Board Member)}

BSAP takes an approach that starts with where people are. Access to food is an immediate need; changing foodways towards health or sustainability are not immediate priorities. Thus, the project sought to provide guidance on healthy food, growing and environmental sustainability through subtle and nonpunitive ways that allow space and time for people to define 'good food' on their own terms and in conversation with one another.

For some, access to food at BSAP provided some breathing space; it is about making budgets go a little further so that a treat may be possible or having the opportunity to try something different or unusual. Local mums reflected on both the challenges and opportunities offered by the FareShare deliveries:

Anne: I know l've got loads of kids, but l've never really bought like a roast? And I got a roast in here, and it was beautiful, aye. And there's other things as well. The burger sauce. Just things that you tend to just walk past in the supermarket you just stick to your own things isn't it.

Stacey: It's expensive sometimes isn't it though.

Lindsey: The things that come in are maybe like $£ 7$, something special. And you're just no going to do it if you've got a big family.

Stacey: You cannae do it.

Lindsey: You buy the smart price, you make it count - you don't want to pay that much for an item. You can't afford this stuff because you've got maybe 5, 6, 7, 8 kids you know.

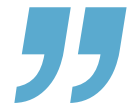

In this sense, the key for BSAP is often about laying groundwork for the possibility of change, rather than working explicitly to change food behaviours. While advice and opportunities to grow and cook are available, they are not compulsory, and the centrality of play and communality to the project means that a broad range of people can access the available support without needing to sign-up to a programme of classes or volunteering.

This broadens the range of users who access the space, from those for whom sustainability and organic food are alien notions to those who are actively thinking about health and food growing. BSAP's strength is in providing an exploratory and non-prescriptive learning space, in contrast perhaps to more formalised programmes for dietary change.

This has benefits in terms of inclusivity and the capacity of BSAP to offer support to a broad range of individuals and families, although it necessarily involves a softer and more implicit impact on diets and sustainable behaviours. 


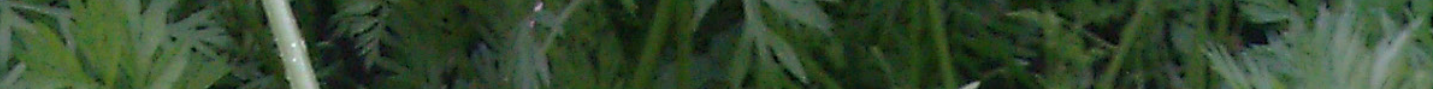

$\mathrm{ar}$

W

as

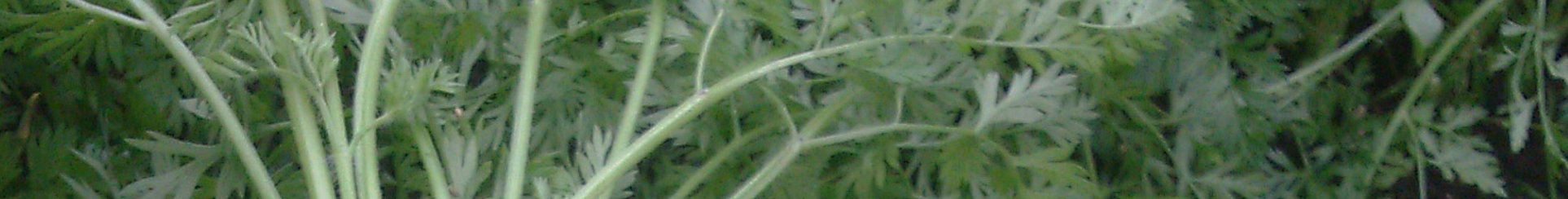

24

y. 1
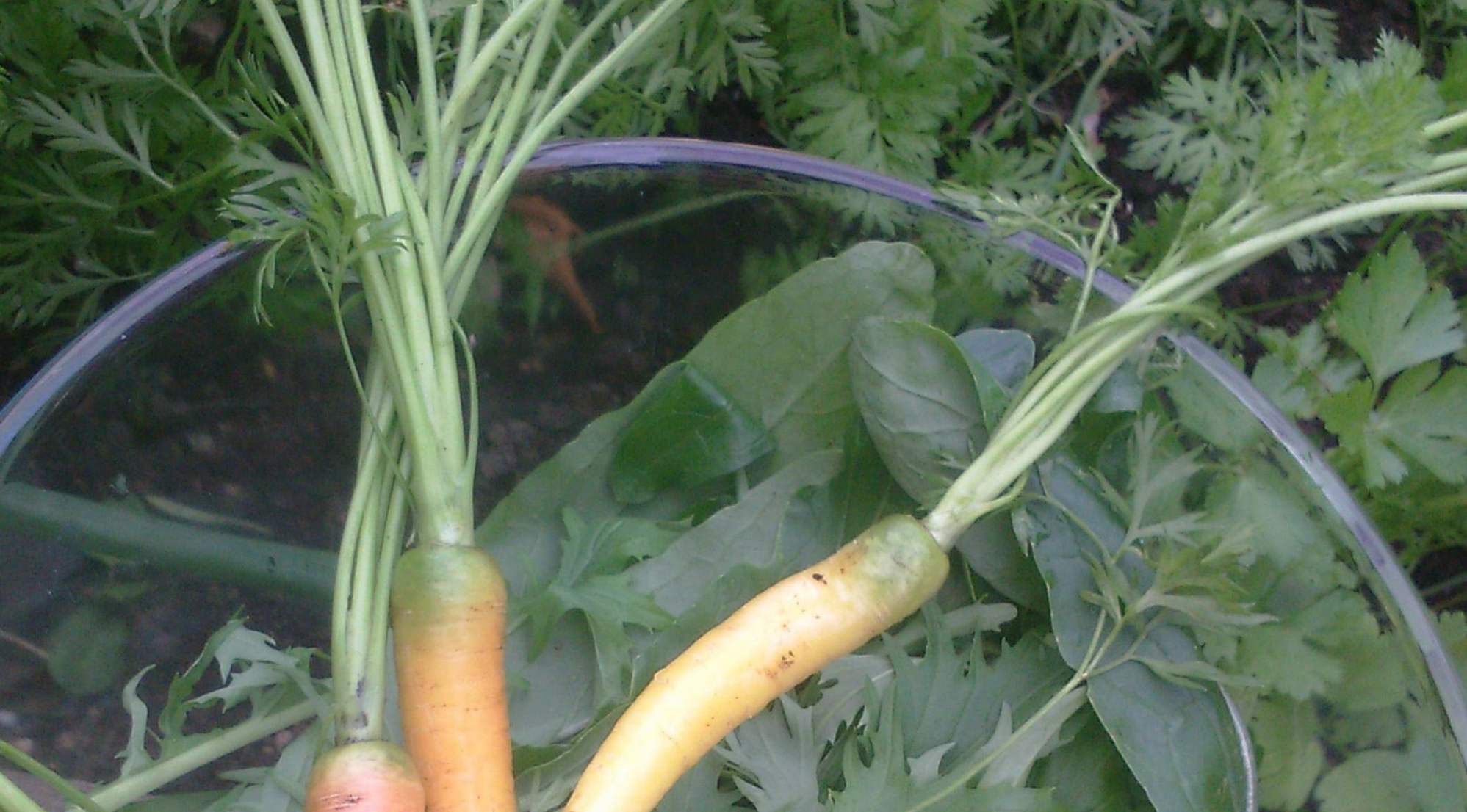

ivi
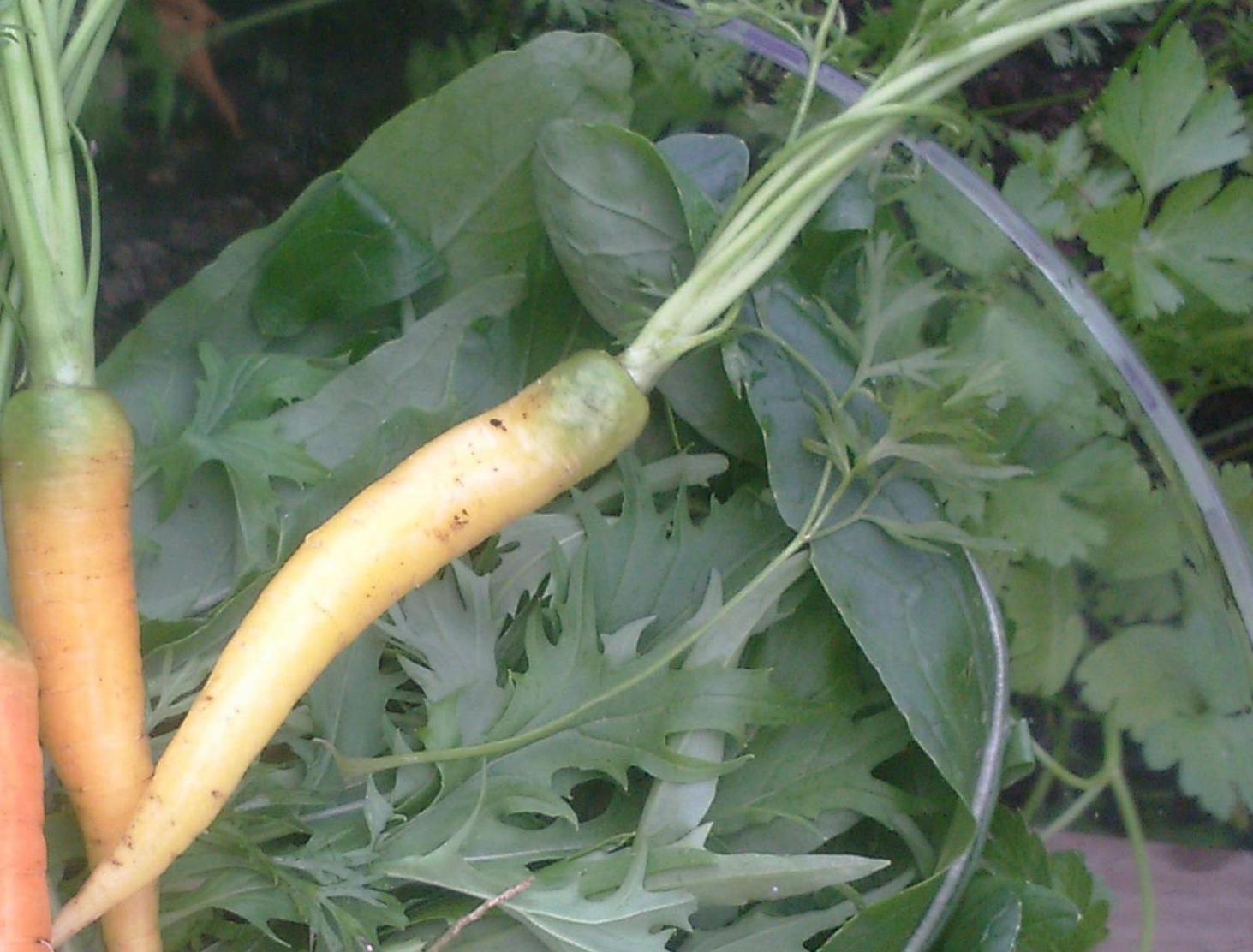

n.

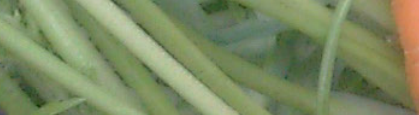

a
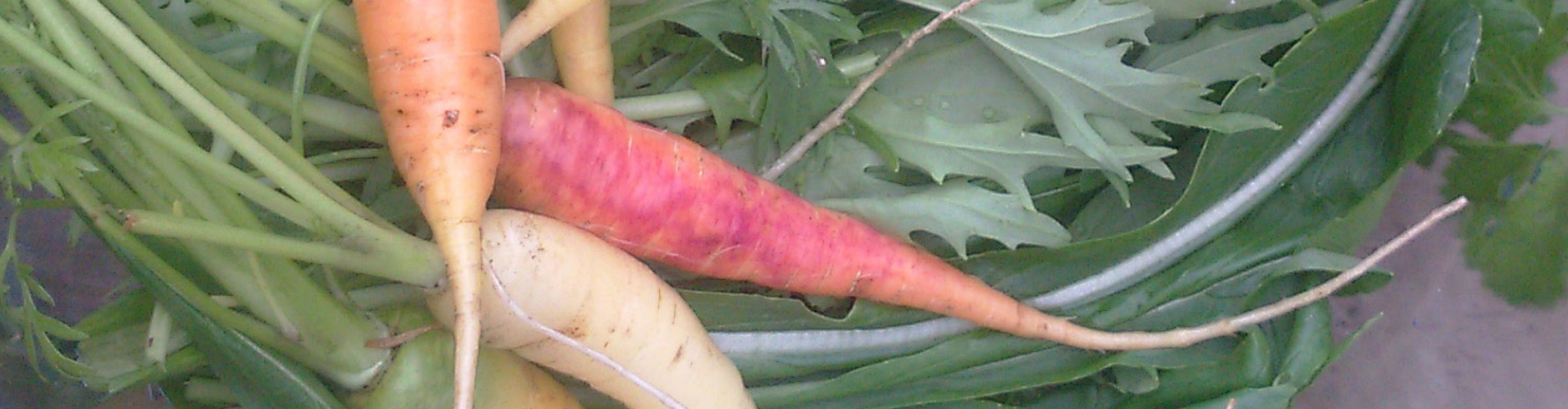


\section{Discussion and Conclusions}

The project has identified two key insights that have emerged in the context of food change and community food provision. The first encompasses the importance of collective ownership and bottom-up visions of sustainability. The second considers sustainability through the lens of urban deprivation. In this sense, it is about recognising differences in capacity that exist within urban areas and the interrelationship of spatial vulnerability and sustainability behaviours.

\section{The importance of community ownership and governance}

BSAP's community focus sits at the heart of its success as a multi-faceted food hub within an area of multiple deprivation. This reflects a broader trajectory that considers the value of building sustainable futures that start not only with community consultation and empowerment, but also in building connections and following the lead of those whose lives are impacted.

\section{Building from joy and the immediate moment}

BSAP start with immediate needs, ensuring that children have 'full bellies' and are having a good time. In taking an approach that allows people to explore food in a relaxed and welcoming way, BSAP has been able to make differences to people's deeply-rooted attitudes towards food and diet.

In this way, BSAP prides itself as a place beyond subsistence, able to feed those who are hungry, but also offering 'treats' and 'giving the kids a choice' (BSAP Playworker 1) about what they can eat. Good nourishing food is not necessarily just healthy or organic food - it can also be food that is satisfying, memorable or special. It is also important at BSAP that those who attend have ownership over their food decisions; rather than having healthfulness or sustainability foisted upon them. In this broad sense, providing good food is at the heart of the BSAP ethic. It is notable that the quality of food provided is of a high standard; often made from scratch, using the best quality ingredients available for the funding the charity gets.

For adults, this might mean being able to access something they have continually passed up because it was over budget and too pricey to take a risk on; for others, it may be connected to a childhood memory. In the same way that children are not 'told' what they can and can't do in the playground, those who engage with the food hub should feel able to simply enjoy the food they are eating and do so in the company of others. Through the fresh food and extra surplus made available through the project, BSAP has been able to engage users in conversations around food and growing that may otherwise not have taken place. 


\section{Facilitating community ownership}

Community ownership of the community food hub activities are important in facilitating the engagement which forms the bedrock of potential food transformations. This was not always straightforward. The garden generated a lot of interest. However, activities were not always accessible or appropriate for the children. Interruptions in participation (such as, during the summer months when the scale of attendance can curtail focused activities) mean that children may not form a sustained relationship with the garden. There remains too a challenge in how to convert passing interest into something more enduring. This is also true for adults, who will often verbalise interest in the garden, but may struggle to commit to regular sessions or be put off by feeling it is not for them.

While the sharing of food can be a practice of equality and foster a sense of community, some barriers do exist, particularly around differing perceptions of need. The association of free food with the stigma and shame of food poverty and food banks cannot be bracketed off from this sense of being needy ${ }^{17}$ and a pride in self-sufficiency. For some, this superseded a willingness to access the free food on offer. This is despite the framing of food as surplus that would otherwise have gone to waste. There are also class and geographical tensions, particularly from those who travel to Dalmarnock from more affluent areas of Glasgow. For example, some middle-class visitors and those bringing children in a care-giving setting (e.g. nurseries and child minders) are sometimes uncertain as to whether the free food is 'for them' so choose not to eat, or offer to make a donation in exchange for their free lunch.

Community ownership of the food hub is important to build further on the activities offered by the project. This includes supporting sustained training and up-skilling of staff and community members, enabling knowledge to be held in the community. The transformative experience of learning about and growing food could be expanded beyond the current project, allowing locals to play an active role in the distribution of food and the support of others locally. Supported by the current project, this has already started via an intergenerational programme at BSAP that allows the children to cook for and distribute meals to older residents, further supporting increased local ownership around food.

\section{Viewing sustainability from the East End of Glasgow}

The collaboration with BSAP generated unique insights in relation to building sustainability from the perspective of a local community as well as the relationship of that local perspective to more macro level sustainability initiatives.

\section{Sustainability from the ground-up}

Adopting BSAP's ethos of 'learning alongside', we were able to better understand BSAP's specific challenges and came to recognise the various ways in which the project aims were already being undertaken or worked towards through BSAP's existing activities and efforts. While sustainability is not an immediate priority for BSAP, there is a longer-term commitment to it and, as such, it is being developed gradually and in a way that appropriately reflects the mission of BSAP and the needs of its community. 
In this way, the project has highlighted the importance of grounded visions of food sustainability that are co-produced with communities, as well as recognising 'green' behaviours already present on-site and through BSAP's wider network, such as, the recycling of white goods, the sharing of meals between neighbours and the re-purposing of junk to make the playground.

Sustainability is, thus, fostered through the deepening of social connections that are facilitated through BSAP. Locals often express a willingness to 'give back' and engage with the social life of BSAP in some way, through volunteering or donating to fundraisers or Christmas gift appeals. As one older female resident put it, 'I feel l'd like to give something back to what they've gave me, you know what I mean and mixing with the community.'

In this way, the support that people receive from the BSAP community food hub may enable them to look out for others in their community, helping to develop new relationships and networks of support and care. BSAP's food work has impacts beyond simply feeding hungry people or providing a pathway to reflect on food behaviours - it also reconnects people with others in a neighbourhood that has experienced the loss of spaces in which to meet neighbours and nurture social connection ${ }^{18}$.

\section{Beyond the local}

Combining a perspective of the community and the vantage point of BSAP affords the potential to consider some broader narratives about sustainability. As one playworker put it, the lives of some locals around BSAP are 'chaotic' (BSAP Playworker 2) and this sometimes lends itself to an understandably narrow focus on 'getting by'. In this way, engaging with recycling, compost or the food system can seem a world away from the immediate concerns of everyday life; leading one local mum of four to note that they had:

Too much else on my plate! Too much else to think about. Em, no, l've never done it. Everything goes in the one bin in my house. It's just far too much else to think about.

Despite the reluctance of this local resident to consider sustainability in the home, this is not to say that sustainability is always alien to the lives of those in Dalmarnock. As noted above, learning with BSAP allowed us to understand how networks of reuse and local support lend themselves to the sustainable use of resources and reduced waste. It is often recognised that those who live with fewer resources have lower carbon footprints than those who consume a great deal of resources through their greater wealth ${ }^{19}$. To this end, it is important to acknowledge that limited engagement with the idea of sustainability as a core message does not necessarily relate to a lower level of lifestyle sustainability. 
Nevertheless, there are aspects of food sustainability, particularly around diet, that if adopted in policy could have harmful impacts on those who live in areas like Dalmarnock. Particularly, one-size-fits-all policies, such as, meat taxes ${ }^{20}$ could have serious consequences in the lives of those whose resources are already constrained and whose access to fresh fruit and vegetables is made difficult by their urban geography. Further, a lack of cultural sensitivity means that those who promote full-scale shifts to vegetarian or vegan diets may overlook the emotional and cultural appeal of 'East End' foods like stovies, a dish of sausage, potatoes and gravy. Such shifts need to be sensitive to the local context and the challenges therein.

There is an increasing recognition of the global readiness for zero carbon economies from activists and scholars ${ }^{21}$, for instance, in setting earlier deadlines for developed economies than for developing economies. Whilst this recognises the capacities of different regions to participate in climate action, we suggest the same should be reflected at a more local level. Viewing sustainability from the perspective of Dalmarnock suggests the need for localised recognition, not only of deprivation, need and capacity to transition but also of sustainability that might go unnoticed, represented as 'thriftiness' or economicallydriven behaviours. Instead of subsuming Glasgow in a homogenised view of a developed city, it is important to recognise the injustices that exist in terms of food access and poverty, and the injustices that might unintentionally accompany top-down visions of sustainability.

Our work with BSAP asks us to consider the kinds of spaces for hope and transformation that are possible within the terrain of urban deprivation, and the ways in which community food can contribute to building sociability and connection, food access and joy and in the longer-term care-full transitions to sustainability. 
University of Glasgow Adam Smith Business School University of Glasgow Gilbert Scott Building Glasgow G12 8QQ

+44(0)1413303993

business-school@glasgow.ac.uk glasgow.ac.uk/business

$f \boldsymbol{y}$ 웅 in Youtube

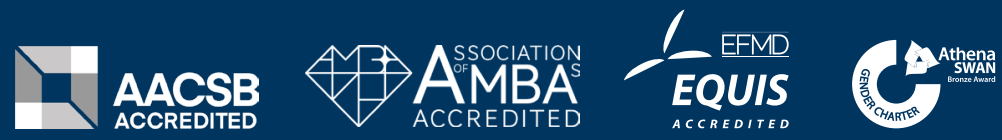

